\section{ABSTRACT OF LECTURES ON THE}

\author{
SURGICAL TREATMENT OF ANEURISM IN ITS \\ VARIOUS FORMS.
}

Dilivered before the Royal College of Surgeons of England.

\author{
By T. II O L MES, F.R.C.S., \\ Professor of Pathology and Surgery.
}

LECTURE I. - Fune 4 th, I872.

Mr. Holmes, after thanking the Council of the College for the honour of his appointment, proceeded to remark upon the great changes which had been made in the surgical treatment of aneurism since this subject had been brought before the College of Surgeons, forty-three years ago, by Mr. Guthrie, and which had necessitated a totally different view of the surgery of this disease from that which he took. Mr. Guthrie's teaching led to the inference that, with few exceptions, aneurisms which are not curable by the Hunterian method are not curable at all. But the success of pressure, as invented by the Dublin surgeons, of the rapid method of pressure under chloroform, of the flexion of the limb in popliteal aneurism, and of digital pressure, has given a new impulse to the search for milder methods of cure in the more accessible forms of aneurism. 'The same success has also led to the conclusion that there are no forms of aneurism, however near the heart, which are in their own nature absolutely incurable ; and has accordingly stimulated surgeons to test the value of sundry plans newly introduced into practice for the cure of aneurisms situated on arteries which are inaccessible to operation. Such are galvano-puncture, distal ligature and distal pressure, manipulation, coagulating injection, the introduction of foreign bodies into the sac. None of these novel plans had as yet proved regularly successful; but it was intended to show, in this course of lectures, that some of them rest on a sound pathological basis, and that further experience will, in all probability, obtain success with them regularly in a certain proportion of cases-though the frequent connexion of internal aneurism with extensive degeneration of the arteries or disease of the heart, forbids us to hope for anything like the success which is obtained in external aneurisms.

The main propositions to be sustained in this course of lectures were announced to be as follows.

I. Aneurisms, of whatever form or however near the heart they may be, ought not to be regarded as incurable, but should be made the objects of definite methodical treatment, internal or external.

2. There is definite proof, from pathological anatomy and from surgical experience, of the curative influence of Brasdor's operation in innominate aneurism, and of its beneficial effects in some cases of aortic aneurism.

3. Arteries may be successfully tied and obliterated without their continuity being interrupted; and this modification of the ligature, whilst affording much security against secondary hæmorrhage, and thus much diminishing the danger of the operation in general, may very probably in future enable surgeons to deal successfully with cases in which it may be necessary to tie the first part of the subclavian (whether on the distal or the proximal side of an aneurism) or the innominate artery.

4. Galvano-puncture may be used with; at any rate, temporary benefit in thoracic aneurism; its use is not so dangerous as to render further trials of it inexpedient; and there is good hope that the method may be so far perfected as to make it a safe and regular plan for the treatment of thoracic, subclavian, and other forms of aneurism.

5. Many cases, such as those in which ligature of the artery near to the heart has been resorted to for the cure of subclavian and subclavio. axillary aneurism, may be made amenable to improved methods of pressure.

6. Aneurismal tumours situated even as high as the lower part of the abdominal aorta, those of the mesenteric and other branches of the aorta, and of the iliac arteries, may be treated with success by rapid coagulation of blood under pressure; but this method is a dangerous one, and should not be used until internal treatment had failed.

7. There are cases of abdominal aneurism in which Mr. Syme's sug. gestion of reviving the old operation is worthy of further trial.

Instances were then adduced of the cure of aneurisms of the aorta by the resources of Nature, and others in which that disease had been cured and the patient restored to health by the enforcement of persistent rest and quiet, more particularly one under Mr. Stanley's care, related by Mr. H. Ludlow in the fifth volume of the Pathological Transactions, and one under the care of Mr. Joliffe Tufnell.* The spontaneous cure of innominate aneurism was also exemplified by a preparation from the Museum of St. George's Hospital.

The various plans of treatment for thoracic aneurism were enumerated. Short mention having been made of the injection of ergotine into the cellular tissue, and of the introduction of iron wire and other foreign bodies into the sac-plans which do not hold out much promise of success-the lecturer proceeded to discuss the internal or medical treatment of internal aneurism. This treatment, a modification of that of Valsalva, had been described by Abernethy and by Mr. Holmes him. self, but has been methodised and brought to perfection by Mr. J. Tufnell. It should be the treatment always first put in practice in any case of internal aneurism, thoracic or abdominal, before the adoption of any more dangerous measures.

Brasdor's operation was then discussed. After a short history of its introduction, Mr. Wardrop's reasoning was considered by what he endeavoured to prove, not only that the operation will succeed where there are no collaterals between the sac and the ligature (which appears indubitable), but also that in innominate aneurism perfect consolidation may be produced by tying the carotid and the third part of the sub. clavian, even though all the four large branches from its first part intervene between the second ligature and the sac. This is opposed to the facts which we know (some of which were instanced) proving the very rapid development of the collateral circulation in man. Mr. Fearn's case + was admitted as proving that sufficient coagulation may follow this operation to produce a practical cure; but the radical cure of the disease, i.e., the entire obliteration of the tumour by laminated coagulum, appears impossible under Wardrop's plan.

Turning from theory to the results of experience, these are twofold, viz., the pathological anatomy of cases where distal impaction of clot has occurred in innominate and aortic aneurism, and the history of cases in which Brasdor's operation has been performed.

The history of the case, previously alluded to, at St. George's Hospital, of spontaneous cure of aneurism of the innominate artery, was detailed, and the preparation (obtained after the man's death, four and a half years later from phthisis) exhibited. Cure had, in this case, been produced by impaction of clot in the carotid artery. This had caused the obliteration of the whole tumour, with the exception of a small channel by which the blood reached the subclavian artery. A drawing was exhibited, taken from a case where the carotid was tied on the distal side of an innominate aneurism, by Dr. Wright of Montreal, which shewed a condition of parts absolutely identical. Mr. S. Lane's and Sir W. Fergusson's cases of distal ligature of the carotid were also adduced in proof of the same proposition, viz., that the distal ligature acts in these cases in exactly the same way as the distal impaction of clot; that its natural (though not necessary) effect is to obliterate the carotid portion of the tumour, and that this may suffice to remove all symptoms of the disease. Again, cases were adduced from the Westminster, the London, and St. Bartholomew's Hospitals to show the effect of distal impaction of clot in the mouth of the subclavian artery, and to prove that, in these cases, the subclavian portion of the sac becomes obliterated, and that the distal ligature of the carotid artery would have almost certainly produced the obliteration of the tumour, and saved the patient's life, which in two of the cases was lost by the bursting of the tumour into the windpipe, and in the third from suffocation. The undeniable inference from these facts is, that Brasdor's operation for innominate aneurism rests on a sound pathological basis.

\section{CASE OF SPINA BIFIDA CURED BY INJECTION.}

BY JAMES MORTON, M.D.,

Surgeon and Lecturer on Clinical Surgery, Glasgow Royal Infirmary.

THE child, who is the subject of the following report, was brought under my notice by Dr. Robert Grieve, of this city, about fourteen days after its birth ; the most prominent part of the tumour was then slightly ulcerated. The mother was directed how to defend it and dress it properly. The following report of it was written by my assistant, Mr. John Caskie.

Ann Ross, aged two months, had a tumour situated over the lower lumbar vertebra. At birth it was small, but had gradually increased in size. Some time ago it showed signs of ulceration; but thesehealed up, leaving thickening and cicatrices of different colours. On admission the tumour was as large as a middle-sized orange, and cylindrical in shape. At some parts it was reddish, and at others bluish in

* In his pamphlet on the Succesful Treatment of Internal Aneurism.
+ The preparation from this case is in the College Museum. 
tint. It was semi-transparent, fluctuant and somewhat wrinkled on the surface. It became tense when the child cried. On looking through it, several striæ were seen passing over its internal surface. On March 27, 1872, it was tapped with a grooved needle, and about three ounces of a fluid resembling cerebro-spinal were drawn off. The child did not suffer ; and, as the fluid continued to exude, the opening (which was made upon the right side towards the top) was closed by means of collodion. On A pril I the child was in fair condition, had no diarrhœa, took the breast well, and was in all respects healthy. On April 2 the tumour was again tapped, and about two ounces of fluid were drawn off. After the tapping the fontanelle was depressed, but by night it was again normal. The child continued well, and no leakage took place. The tumour was dressed with a small piece of oiled lint. On April 5 the tumour was tapped with a small trocar and cannula, and half its contents were drawn off. About two drachms of a solution of iodine in glycerine were slowly injected, and the tumour was dressed with oiled lint. The child suffered a little from shock at first, but soon recovered. It continued well during the day, taking the breast well. The fontanelle was for a time depressed. At night the tumour was half filled, and the fontanelle was nearly normal. The lower limbs on examination were found to be quite normal, as regarded colour and temperature. On April 6 th, the tumour was about three-fourths of its former size. The contents were thicker; but no distinct coagulation had taken place. The child continued well. On the 8th, slight ulceration was observed over the top of the tumour. It was diminished in bulk, and was now only about half its former size. The child had complete power over the lower limbs, and was well. The tumour continued to diminish; and on the 16 th consolidation had taken place at the upper portion, and the other parts felt firmer than formerly. The ulceration had not increased, but there was slight discharge of pus from under the seat that covered it. On the $25^{\text {th }}$ the tumour was nearly on a level with the surrounding skin, and only distinguishable from it by its livid colour. Over its centre there was a small triangular depression, from which a small slough was taken by Dr. Morton. The child was in all respects healthy, and had complete power over its limbs.

On the 6th April last, in the British Medical Journal, there is an account of the first and only other case which I have treated in the manner here noted, so that both have been successful. The composition of the iodine solution will also be found there stated.

The procedure adopted may be shortly re-stated thus. I. Two tentative tappings are made with a grooved needle, with an interval of four or five days between the two. 2. The tumour is tapped with a small trocar and cannula, allowing about half the contents of the sac to escape, and about a drachm of the iodine solution is injected; rather more was used in this case, but, I think, less might have sufficed. The aftertreatment may be said to consist in dressing with some bland substance as oil or lard, cleanliness and care, so as to avoid local injury or irritation.

Prevention of the complete escape of the cerebro-spinal fuid I believe to be of the greatest importance ; hence the use of collodion in this case, which answered the purpose admirably. The presence of this fluid is essential to the functional, if not the structural, integrity of the spinal cord and brain, and when it is allowed to drain away the child speedily succumbs. On this point I can speak from some experience; besides, soon after treating the case already published, a child was sent into the infirmary having a similar tumour in the dorsal region, which was punctured by the grooved needle, but not injected; the spinal fluid continued to exude, and from this alone the child sank and died. The idea of using the collodion did not occur to me till too late, but this mishap caused me to direct my assistant to watch the case now given, and use the collodion if necessary, and it forms an apt illustration of the success of such a mode of closing the puncture. After the second puncture the collodion was not required.

To my mind, the satisfaction attending the success of this case is enhanced by the circumstance of its being the second of two cases treated in precisely the same way; and these the only two that have been treated by injection with the glycerine solution, which I was induced to adopt, as being less diffusible than either a watery or spirituous solution.

How far this mode of treatment may be applicable to the cases in which the congenital defect exists above the lumbar region, I will not at present endeavour to define ; but surely it is not too much to say that if by it we can save lives, when we have a lumbar tumour to deal with, we are not to be debarred from attempting to deal with those in the dorsal or cervical region, provided the extension of our line of operations be prudently conducted.*

* This case was made the subject of some clinical remarks; and the child was shown to the Glasgow Medico-Chirurgical Society on the evening of May 3rd.

\section{ON THE CAUSATION AND SIGNIFICANCE OF THE CHOKED DISC IN INTRA- CRANIAL DISEASES.}

\author{
BY W. H. BROADBENT, M.D., F.R.C.P., \\ Physician to St. Mary's Hospital.
}

THE ophthalmoscope, thanks to the efforts of Dr. Clifford Allbutt and Dr. Hughlings Jackson, may be said fairly to have taken its place in English medicine; and it is scarcely possible to overstate the additional interest it has brought into the study of the diseases of the nervous system. It has removed causes of confusion and error; it has opened absolutely new sources of information as to the state of the intracranial circulation, and has thus given new zest to the pursuit of precision in diagnosis, which will eventually conduce greatly to decision in treatment. It has also, or rather the far-reaching vision of the men who have forced it upon the attention of the profession, widened the horizon of pathological research by stating new questions of various kinds for solution.

The problem of the causation and significance of the choked disc in intracranial diseases is one of primary importance. It has been most ably treated by Dr. Allbutt in his work on the Ophthalmoscope; but all who are patiently endeavouring to follow in his footsteps will be glad that he has returned to the subject and discussed it in the columns of the JOU RNAL, affording an opportunity for stating difficulties and seeking the special information needed by different individuals. It may also elicit the experience of other observers, and possibly some contributions of value. I think a difficulty very generally experienced is the discrimination of the choked disc. This difficulty is mentioned by Dr. Allbutt in his communication, and is attributed to coexistence of congestion-papilla and neuritis proper. Either, however, these mixed forms of affections of the nerve and retina are disproportionately frequent, or I personally fail to estimate fully the distinctions. No doubt this is in some measure due to the fact that I have only very imperfectly mastered the direct method of ophthalmoscopic examination, and have been unable to bring it to bear in the cases of meningitis in which my uncertainty has arisen. The one point with respect to which I most distrust my observations or inferences is as to the elevation or swelling of the disc. I had in several cases concluded, from the appearances seen, that this swelling was present. The retinal vessels have seemed to emerge from a depression in the centre of the elevated disc, to run down a slope and curve from its base to the retina. A slightly varying focussing of the lens has been required to see them with equal clearness in the different parts of their course. With this, the proper vessels of the disc have been visible, and its margin imperfectly defined; and yet, after death, no elevation of the disc existed. In all cases, I have removed the back of the eye myself in the way recommended by Dr. Allbutt, and have carefully avoided traction on the nerve, lest the appearance which I desired to see might be disturbed. I should have been disposed to think that the swelling disappeared at death, like the redness of the choroid, had not the contrary been found.

Accepting, as I think we must, after the confirmation afforded by Dr. Allbutt's prolonged and careful investigation, the distinction drawn by Von Gräfe between the choked disc due to obstructed venous return and neuritis propagated by continuity along the nerve from the meninges, the questions as to the causation of the former condition appear to be - I. Whether simple venous obstruction, with or without the multiplying action of the sclerotic ring, is an adequate cause; 2 . Whether general increased intracranial pressure, producing its effects in the direction of least resistance, may not be, at any rate, one mode of causation; and, if so, whether differences in the origin of the compression, intraventricular or extraventricular, etc., and consequent difference in the direction of the compressing force, may not have some in fluence in the production or non-production of this particular result. 3. Whether distension by fluid of the lymph-space between the outer and inner sheath of the nerve may be a link in the causation by compression of the nerve-bundles and vessels. This is a new point raised by Dr. Allbutt; and the further question arises-how the effusion in the nerve-sheath is produced.

I do not propose to discuss in detail these possible modes of causation. The first is acknowledged to be insufficient to account for all cases. The second is the one which best satisfies my judgment ; and I have adopted it as a provisional hypothesis and basis for investigation, the establishment or overthrow of which cannot fail to be instructive, and to bring us one step nearer to the true explanation. For the present, I wish simply to contribute some observations bearing on this and on the third question-the influence of hydrops vaginæ, an ex- 